\title{
Erratum to "Designing graphene based nanofoams with nonlinear auxetic and anisotropic mechanical properties under tension or compression" [Carbon 111 (January 2017) 796-806]
}

\author{
Andrea Pedrielli a, b , Simone Taioli b, c , Giovanni Garberoglio b, ${ }^{*}$, Nicola M. Pugno a, d, e, ${ }^{* *}$ \\ a Laboratory of Bio-inspired \& Graphene Nanomechanics, Department of Civil, Environmental and Mechanical \\ Engineering, University of Trento, Via Mesiano 77, 38123, Trento, Italy \\ ${ }^{b}$ European Center for Theoretical Studies in Nuclear Physics and Related Areas (ECT*-FBK) and Trento Institute for \\ Fundamental Physics and Applications (TIFPA-INFN), 38123, Trento, Italy \\ ${ }^{c}$ Faculty of Mathematics and Physics, Charles University, 180 00, Prague 8, Czechia \\ ${ }^{a}$ Center for Materials and Microsystems, Fondazione Bruno Kessler, Via Sommarive 18, 38123, Povo, TN, Italy \\ e School of Engineering and Materials Science, Materials Research Institute, Queen Mary University of London, London, \\ E1 4NS, UK
}

The publisher regrets that the Graphical Abstract was not updated and the Supplementary material was not uploaded.

The publisher would like to apologise for any inconvenience caused.

Appendix A.

Supplementary data

Supplementary data related to this article can be found at http://dx.doi.org/10.1016/j.carbon.2017.01.064.

mmc1.docx: Unit_Cell_A.docx

mmc2.docx: Unit_Cell_B.docx

mmc3.docx: Unit_Cell_C.docx

mmc4.docx: Unit_Cell_D.docx 